\title{
Perspectives of Photonics Technology to Diagnosis COVID-19 Viruses: A Short Review
}

\author{
Bakr Ahmed Taha \\ UKM-Department of Electrical, Electronic and Systems Engineering, Faculty of Engineering and Built \\ Environment, Universiti Kebangsaan Malaysia, UKM Bangi 43600, Malaysia ${ }^{1}$
}

\author{
Article information \\ Article history: \\ Received: April, 12, 2021 \\ Accepted: April, 22, 2021 \\ Available online: April, 26, 2021 \\ Keywords: \\ Photonics \\ COVID -19 \\ Laser diagnosis \\ Coronavirus \\ Optical biosensor \\ Correspondence: \\ Bakr A. Taha \\ p103537@siswa.ukm.edu.my
}

\begin{abstract}
The COVID-19 disease outbreak has emphasized the critical need for more sensitive analytical technology. Photonic technology focuses on studying light interaction analysis with the molecules to enhance diagnostic tools' accuracy. Due to the distinct spectral signatures, lasers have shown effectiveness in the classification and monitoring of viruses. This work aims to improve healthcare delivery in public areas, markets, hospitals, and airports. However, providing insights into the technical aspect also helping researchers identify the possibilities and difficulties in this field. This short review has been collect from four authoritative databases: Web of Science, Science Direct, Scopus, Google Scholar. This paper discusses emerging developments in photonic sensor applications such as telehealth, point care, and telescreens in environmental surveillance. It also includes modern studies to identify and diagnose viruses by using photonic techniques. Finally, it was found that the most effective approaches for reducing the spread of the COVID-19 virus pandemic in the environment, besides collecting the big data via an intelligent optical fibre network between the hospitals and other public places.
\end{abstract}

DOI: 10.53293/jasn.2021.11016, Department of Applied Science, University of Technology

This is an open access article under the CC BY 4.0 license.

\section{Introduction}

Photonics is known as the science of generating energy, detecting, and transmitting information using light. Another way to describe photonics is as the science and technology of producing and harnessing light and various other sources of radiant energy whose quantum unit is the photon. Also, permeates almost every part of life, from microscopy to optical communications. As well, involves cutting-edge uses of lasers, optics, fiber-optics, and electrooptical devices in numerous and diverse fields of technology-alternate energy, manufacturing, health care, telecommunication, environmental monitoring, homeland security, aerospace, solid-state lighting, and many others. As a result, when faced with global pandemic threats, it's unsurprising that many solutions have been discovered and created by optical engineering in photonic systems[1-3]. Thus, photonics researchers and companies have made significant contributions to diagnostics and personal protection equipment through integrating advanced systems and creating revolutionary developments in this area. Coronaviruses have been reported for too many health problems over the last two decades. According to WHO info, the COVID-19 viruses considered a higher purpose death rate (approximately 3.3 percent) than the prior influenza pandemics in 1918 and 1957, and its dissemination rate was even 40 times $[4,5]$. Resulting from COVID -19 virus, nearly 139 million people infected and caused about three million deaths, according to the last report of the World Health Organization. 


\subsection{Emergence and characteristics of SARS- CoV-2.}

Coronaviruses family are a complex collection of viruses and can cause moderate to severe respiratory infections in humans and animals as shown in figure (1). Two types of zoonotic-coronaviral high pathogens, such as SARS-CoV and MERS-CoV, occurred and caused lethal breathing disease in humans in 2002 and 2012 and became a new public health issue the 21st century. Evolving coronaviruses became a significant concern of the public health community [6]. Several health facilities in Wuhan, Hubei province, China, registered clusters of patients with pneumonia of unknown cause in late December 2019 [7]. Coronaviruses groups are classified into four genera: Alpha-coronavirus, Beta-coronavirus, Gamma-coronavirus, and Delta-coronavirus [8]. The structure of COVID-19 viruses' shape consisted of four parts are spike $(\mathrm{S})$, membrane $(\mathrm{M})$, envelope $(\mathrm{E})$, and nucleocapsid $(\mathrm{N})$. It has the largest genome between 26.4-31.7 $\mathrm{kb}$ of all recognized RNA viruses. Besides, the genetic material of the virus included singlestranded RNA [9],[10].

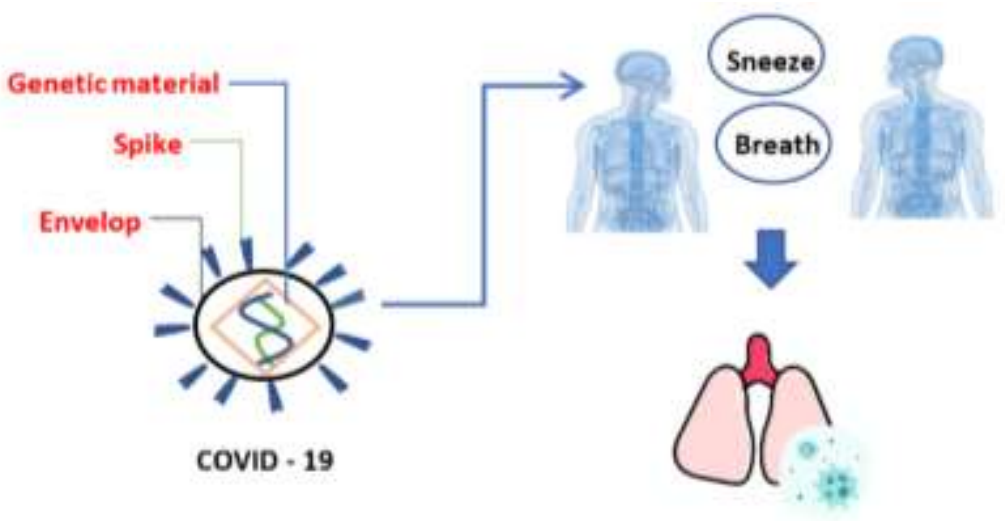

Figure 1. Illustration of a feature shape COVID-19 virus and human transmission exchange

\subsection{Background}

The application of nanomaterials with photonics in biosensing can solve many of the problems and limitations of biosensor technology. Nanomaterials are describing as having dimensions between 1 and $100 \mathrm{~nm}$. They have designed to exhibit novel properties compared to materials lacking nanoscale features, such as improved strength and conductivity and unique optical, magnetic, thermal, and chemical properties. Thus, dependent on affinity, integrate the high sensitivity of optical biosensors tools, specific receptors [11]. The researchers, a dual-functional plasmonic biosensor using the plasmonic photothermal (PPT) effect in conjunction with localized surface plasmon resonance (LSPR), have developed a COVID-19 diagnosis. The methods were used two-dimensional gold Nano islands integrate with an optical biosensor as a platform. As a result, the experiment achieved a high sensitivity at a lower detection limit of $0.22 \mathrm{pM}$. [12]. A Study shows the SARS-CoV-2 identification using the Raman spectroscopy analysis method to detect a SARS-CoV-2 virus in saliva, and the results were accuracy of $91.6 \%$, sensitivity 92.5 $\%$, and specificity $88.8 \%$ [13]. Femtosecond pulse lasers emitting near-infrared have been used to inactivate many viruses with no damage to human cells [14],[15]. The fibro-optic absorbance platform (P-FAB) biosensor was proposed to identifying the SARS-CoV-2 virus without pre-processing in the patient's saliva specimen [16]. A new biosensor based on field-effect transistor (FET) is reported for diagnostic SARS-CoV-2 in clinical samples. Graphene sheets are coating with a particular antibody against SARS-CoV-2 spike protein. The limit of detection was 1 and $100 \mathrm{fg} / \mathrm{mL}$ [17]. An optical fiber sensor device based on Evanescent Wave Absorbance (EWA) to detect COVID-19 viruses is developed via measuring the refractive index variations at light propagated inside the optical fiber [18]. This review focuses on the innovative methods to fight the COVID-19 virus based on photonics technology in literature studies. Micro - cavities have considered a leading method, drawing a broad interest in improving lightmatter interactions in biomedical and biomedical fields[19].

\section{Identification COVID - 19 via photonics.}

Viruses are the smallest molecules of pathogens known. However, most of them cause significant deterioration of human health. Most of the time, the host's natural immune defense mechanism is the best-known treatment for 
viruses; Otherwise, the only alternative is the initial avoidance of viral infection. Therefore, diagnosis is the direct approach against the overall target of monitoring and removal of viruses.

\subsection{Laser spectrum Interaction with Molecules}

Interactions between light and matter make a significant contribution in many scientific areas, creating critical spectroscopy applications, sensing, quantum information extraction, and lasers. Light waves spread at the speed of light in a vacuum, for most of these purposes. Matter consists of charged particles because atoms have a positively charged nucleus and spin around the negative charge electrons. The electric field changes with time whenever charges are in motion. The light is considered as electro-magnetic waves and described as a stream of particles called photons. Each photon carries a ratio of energy, matter can absorb photon energy, and spectroscopy is the science that analyzes the light emitted value. Light is categorized according to its spectrum of wavelengths. When reacting with materials, it can cause ionization effects, according to the ascending order of wavelengths such as X-rays, ultraviolet rays, visible light, infrared rays, microwaves, and gamma rays. The smaller the wavelength, the higher the frequency, and the higher the energy. It can induce different phenomena when it interacts with matter because of the electromagnetic properties of light. Otherwise, when light of $100 \mathrm{~nm}$ wavelength interacts with a substance, it will cause the material atoms to ionize. As the wavelength increases, the light wave's energy is not enough to cause ionization. Figure (2). Shows the comparison of the size of viruses for selecting wavelength suitable used to diagnose COVID - 19 viruses.

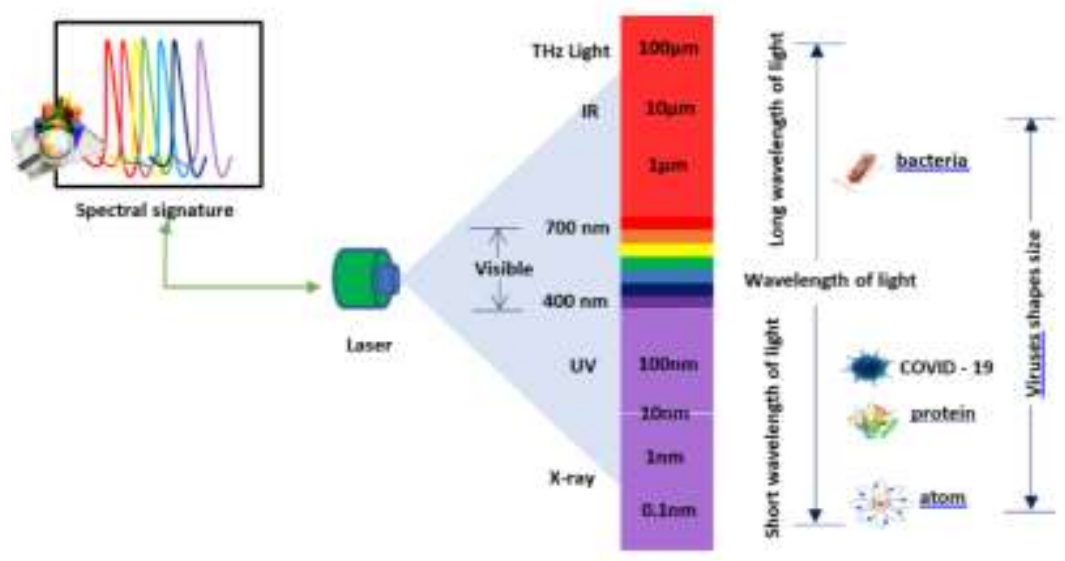

Figure 2. Comparison of the size of viruses for selecting wavelength suitable used to diagnose COVID - 19 viruses.

When light is incident on an atom, three necessary processes happen: absorption, spontaneous emission, and stimulated emission by which light interacts with particles. Depending on the type of matter, absorption occurs while the atom is a lower energy level, the electron atom lifts it to the upper energy level by consuming the energy of photon creating up and down transitions between energy levels that called spontaneous emission. The electron will be in an upper energy level (E2) or excited state and spontaneously decays to a lower energy level (E1) and radiates a photon light. The emitted photons contain energies peculiar to that substance, as each material has a particular collection of energy levels. The frequency $(v)$ and wavelength $(\lambda)$ of the light are related to these photon energies by:

$$
\Delta \mathrm{E}=\mathrm{hv}=\frac{h c}{\lambda}
$$

Where $\mathrm{h}$ is a constant for Planck and $\mathrm{c}$ is the speed of light. The effectiveness of the laser application tool in Covid19 battle has many applications such as remote monitoring, tele-screening/telehealth, intelligent network \& big data, improving environmental quality, and improving the quality of foods.

\subsection{Keys to diagnose viruses.}

In this section, the essential keys for the detection of the viruses have been described. The intensity Isc of the scattered light can be described as the following function of five variables:

$$
I s c=\operatorname{Isc}(c, d, \theta, \lambda, n)
$$

Where $c$ is the concentration, $d$ the particle diameter, $\theta$ the measuring angle, $\lambda$ wavelength of the light, and $n$ the refractive index of the particles relative to the surrounding medium, the calculation of this equation is part of 
scattering theory. Variables affecting light scattering such as wavelength, particle size, refractive index, angle of incident, and concentration [20], are as shown in the schematic in figure (3).

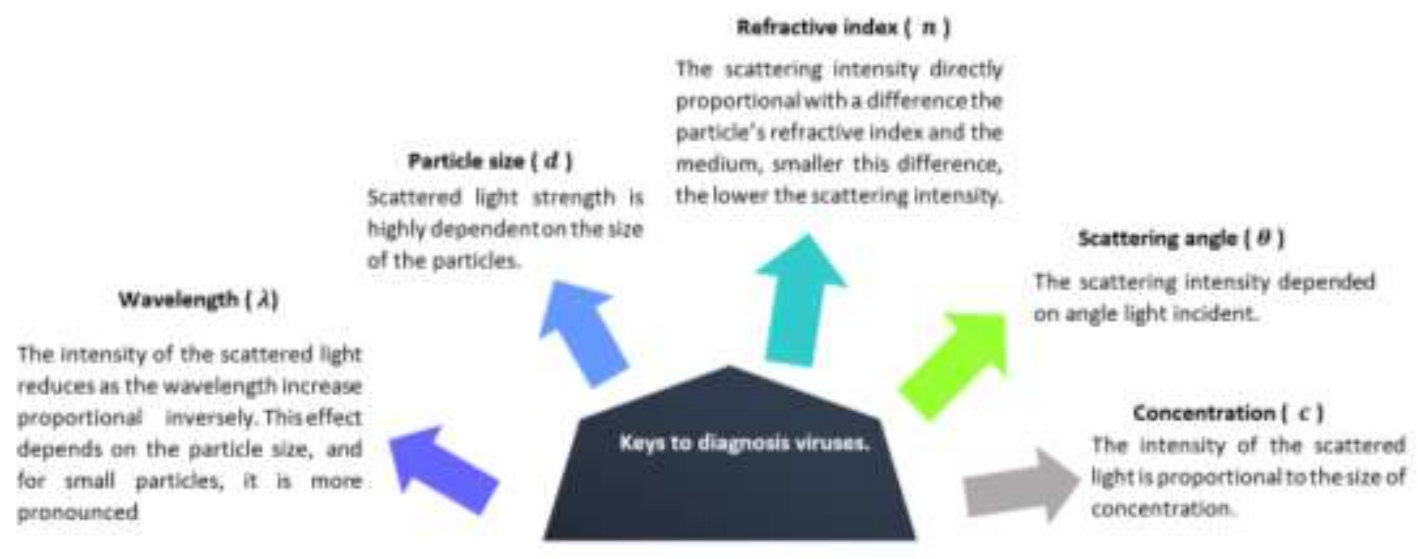

Figure 3. A schematic description of parameters affecting light scattering

\section{Lasers diagnosis of viruses.}

This section describes the diagnosis and classification of the viruses by using laser techniques, such as Laser-induced fluorescence-light detection and ranging (LIF- LiDAR), Quantum cascade lasers (QCLs), and Surface-enhanced Raman spectroscopy (SERS). Light detection and ranging are a category of methods focused on the principle that when a pulsed laser impinges on particles, the particles absorb and emit signals that can be characterized using a suitable detector [21]. LiDAR systems are used to identify and characterize biogenic materials, for example, pathogenic bacteria in both laboratory and field environments [22]. A study of the Laser-induced fluorescence-light detection and ranging (LIF- LiDAR) technique has shown that it can be used for detection, real-time monitoring, and classification of viruses in various environments [23]. A significant benefit of the LIF-LiDAR is the long-range detection of dangerous bio-aerosols, particularly those classified as biological weapons. Nevertheless, selecting the accurate wavelength for stand-off investigation of viruses in the atmosphere faces some difficulties.

Quantum cascade lasers (QCL) are the first room-temperature semiconductor laser source for the mid-IR spectral field. QCLs are categorized based on the resonator configuration to the three popular sorts of laser resonators such as Fabry Perot (FP), Distributed Feedback (DFB), and External Cavity (EC). It employs a spectrum signature of long-wave infrared (LWIR) reflectance to detect nanoparticle chemicals on contaminated surfaces using a quantum-cascade laser (QCL) that tunes from $\lambda=9.1$ to $9.8 \mu \mathrm{m}$ [24][25]. Enhancement of mid-infrared spectroscopy methods based on Fourier transform infrared (FTIR) and quantum cascade lasers (QCLs)can be used for environmental monitoring [26]. This model is beneficial because it provides the potential to track a wide variety of high-sensitivity gases to extended ranges. Additionally, the system can be used-perform spectroscopy in transmission mode with a gas cell or in reflection mode for detecting contaminants on surfaces. Also, the device does not need consumables, resulting in low downtime relative to a point sensor network. It is also suitable for the monitoring of large indoor areas such as airports. Spectroscopy is the method that analyzes the optical spectrum produced by the material due to its interaction with light. COVID-19 virus detection using the Surface-enhanced Raman spectroscopy (SERS) technique have been proposed due to its ability to recognize unique gene sequences and it does not require a long time for DNA amplification of COVID-19 [27]. The SERS technique can also be used to identify food contamination [28]. It is an impressive technique that can also identify the molecular fingerprint spectrum with ultrahigh sensitivity. Great successes have been achieved for the sensitive and specific detection of biomolecules, such as pathogens, cancer cells, cellular processes, etc. However, it has weak selective isolation of homologs and components with similar structural properties.

A study reported a new technique for quickly visualizing the droplets emitted while speaking and using different masks' efficiency in reducing their spread. The methodology used a cheap visible laser to imaging particles pass through scattering light [29]. Table (1) shown literature studies laser potential for detecting viruses. 
Table 1. Studies of Laser diagnosis of viruses summarized.

\begin{tabular}{|c|c|c|c|c|c|}
\hline Techniques & Waveleneth & $\begin{array}{l}\text { Spectrum } \\
\text { classify }\end{array}$ & Target & Chullenges & Net \\
\hline UFUUDAR & $\begin{array}{c}248-294 \\
\mathrm{~nm}\end{array}$ & UV range & Hiosernol vinutes & $\begin{array}{l}\text { - The cancentration of virutes in the } \\
\text { atmosohere, indoor ait, or surfates. } \\
\text { - Boaeracol monitoring neets high pulsed inser } \\
\text { excitation } \\
\text { - high cont. } \\
\text { - selection of wavelength. }\end{array}$ & |22] \\
\hline qai & $\begin{array}{l}9.1 \text { to } 9.8 \\
\operatorname{um} \\
8 \text { ta } 14 \\
\mathrm{um}\end{array}$ & All range & $\begin{array}{l}\text { Chemical easer } \\
\text { and, Bacteria an } \\
\text { Surface }\end{array}$ & $\begin{array}{l}\text { - The received spectrum distorted and mived } \\
\text { have appears. }\end{array}$ & [24],|25] \\
\hline Sens & $\begin{array}{l}\mathrm{s30} \mathrm{nm,785} \\
\mathrm{nm}, 532 \mathrm{~nm}\end{array}$ & $\begin{array}{l}\text { In, visible } \\
\text { range }\end{array}$ & $\begin{array}{c}\text { DNA of covid - } 19 \\
\text { virises }\end{array}$ & $\begin{array}{l}\text { - low sensitivity } \\
\text { - multiplex detiection problems inherent to } \\
\text { fluorescence delection } \\
\text { - weak selective isolatian of homologs. } \\
\text { - Components with similar structural properties. }\end{array}$ & {$[26,[27]$} \\
\hline $\begin{array}{l}\text { Laser } \\
\text { scatterine }\end{array}$ & $532 \mathrm{~nm}$ & Visitile & Masks efficiency & $\begin{array}{l}\text { - A small sampie. } \\
\text { - Sire of a droplet. } \\
\text { - The rufractive inder of the droplet. }\end{array}$ & [25] \\
\hline
\end{tabular}

\section{Analysis outcome of literature review}

The COVID-19 pandemic has shown the challenges of existing most analytical technologies for biological detection. However, it need to emphasize developing new, improved diagnosis methods. Photonics are powerful techniques for detecting viruses because the laser's properties can classify and identify viruses according to wavelength emission like a spectrum signature. In addition, it is possible collect big data from any probe laser through a fibre optics network to enhance healthcare quality.

\section{Conclusion}

COVID-19's global pandemic had a significant impact on human life. Worldwide, there has been a noticeable rise in the number of people infected; various companies, governments, and academics are attempting to respond to this global emergency. This short review summarizes the Appearance and Features of SARS-CoV-2 and understanding of advanced photonics systems based on laser and optical fibre sensors application. Also mentioned challenges with a viewpoint. As can be seen, in prospect, laser-based optical detection imaging could aid in environmental monitoring, virus concentration analysis in the air, and food quality testing. Thus, data can be collected for COVID19 monitoring through an intelligent network of medical biosensors, thereby improving healthcare quality.

\section{References}

[1] W. R. Zipfel, R. M. Williams, and W. W. Webb, "Nonlinear magic: Multiphoton microscopy in the biosciences," Nature Biotechnology, vol. 21, no. 11, pp. 1369-1377, 2003, doi: 10.1038/nbt899.

[2] A. Kovach et al., "Emerging material systems for integrated optical Kerr frequency combs," arXiv, pp. 1-84, 2020, doi: 10.1364/aop.376924.

[3] J. Lukose, S. Chidangil, and S. D. George, "Optical technologies for the detection of viruses like COVID-19: Progress and prospects," Biosensors and Bioelectronics, vol. 178, no. January, p. 113004, 2021, doi: 10.1016/j.bios.2021.113004.

[4] World Health Organization, "Laboratory testing for coronavirus disease 2019 (COVID-19) in suspected human cases," no. March, pp. 1-7, 2020.

[5] B. Gates, "Responding to Covid-19 - A Once-in-a-Century Pandemic," pp. 2020-2022, 2020.

[6] J. Cui, F. Li, and Z. L. Shi, "Origin and evolution of pathogenic coronaviruses," Nature Reviews Microbiology, vol. 17, no. 3, pp. 181-192, 2019, doi: 10.1038/s41579-018-0118-9.

[7] N. Zhu et al., "A Novel Coronavirus from Patients with Pneumonia in China, 2019," New England Journal of Medicine, vol. 382, no. 8, pp. 727-733, 2020, doi: 10.1056/nejmoa2001017.

[8] M. Yang, H. Li, J. Sun, Y. Zhao, and D. Tang, "Focus on Characteristics of COVID-19 with the Special Reference to the Impact of COVID-19 on the Urogenital System," Current Urology, pp. 79-84, 2020, doi: $10.1159 / 000499255$.

[9] L. Mousavizadeh and S. Ghasemi, "Genotype and phenotype of COVID-19: Their roles in pathogenesis," Journal of Microbiology, Immunology and Infection, pp. 0-4, 2020, doi: 10.1016/j.jmii.2020.03.022. 
[10] B. A. Taha, Y. Al Mashhadany, M. H. H. Mokhtar, M. S. D. Bin Zan, and N. Arsad, "An analysis review of detection coronavirus disease 2019 (Covid-19) based on biosensor application," Sensors (Switzerland), vol. 20, no. 23, pp. 1-29, 2020, doi: 10.3390/s20236764.

[11] R. Antiochia, "Nanobiosensors as new diagnostic tools for SARS, MERS and COVID-19: from past to perspectives," Microchimica Acta, vol. 187, no. 12, 2020, doi: 10.1007/s00604-020-04615-x.

[12] G. Qiu, Z. Gai, Y. Tao, J. Schmitt, G. A. Kullak-Ublick, and J. Wang, "Dual-Functional Plasmonic Photothermal Biosensors for Highly Accurate Severe Acute Respiratory Syndrome Coronavirus 2 Detection," ACS Nano, vol. 14, no. 5, pp. 5268-5277, 2020, doi: 10.1021/acsnano.0c02439.

[13] S. Desai et al., "Raman spectroscopy-based detection of RNA viruses in saliva: A preliminary report," Journal of Biophotonics, vol. 13, no. 10, pp. 1-5, 2020, doi: 10.1002/jbio.202000189.

[14] K. T. Tsen, S. W. D. Tsen, C. L. Chang, C. F. Hung, T. C. Wu, and J. G. Kiang, "Inactivation of viruses by coherent excitations with a low power visible femtosecond laser," Virology Journal, vol. 4, pp. 1-5, 2007, doi: 10.1186/1743-422X-4-50.

[15] K.-T. Tsen et al., "Photonic approach to the selective inactivation of viruses with a near-infrared subpicosecond fiber laser," Journal of Biomedical Optics, vol. 14, no. 6, p. 064042, 2009, doi: 10.1117/1.3275477.

[16] D. Murugan, H. Bhatia, V. V. R. Sai, and J. Satija, "P-FAB: A Fiber-Optic Biosensor Device for Rapid Detection of COVID-19," Transactions of the Indian National Academy of Engineering, vol. 5, no. 2, pp. 211-215, 2020, doi: 10.1007/s41403-020-00122-w.

[17] G. Seo et al., "Rapid Detection of COVID-19 Causative Virus (SARS-CoV-2) in Human Nasopharyngeal Swab Specimens Using Field-Effect Transistor-Based Biosensor," ACS nano, vol. 14, no. 4, pp. 5135-5142, 2020, doi: 10.1021/acsnano.0c02823.

[18] P. Nag, K. Sadani, and S. Mukherji, “Optical Fiber Sensors for Rapid Screening of COVID-19," Transactions of the Indian National Academy of Engineering, vol. 5, no. 2, pp. 233-236, 2020, doi: 10.1007/s41403-020-00128-4.

[19] X. Fan and S. H. Yun, "The potential of optofluidic biolasers," Nature Methods, vol. 11, no. 2, pp. 141-147, 2014, doi: $10.1038 /$ nmeth.2805.

[20] E. Huber and M. Frost, "Light scattering by small particles," Journal of Water Supply: Research and Technology - AQUA, vol. 47, no. 2, pp. 87-94, 1998, doi: 10.1046/j.1365-2087.1998.00086.x.

[21] S. Buteau et al., "Bioaerosol standoff monitoring using intensified range-gated laser-induced fluorescence spectroscopy," Advanced Environmental Monitoring, pp. 203-216, 2008, doi: 10.1007/978-1-4020-6364-0_16.

[22] C. S. Ho et al., "Rapid identification of pathogenic bacteria using Raman spectroscopy and deep learning," Nature Communications, vol. 10, no. 1, 2019, doi: 10.1038/s41467-019-12898-9.

[23] O. Owoicho, C. O. Olwal, and O. Quaye, "Potential of laser-induced fluorescence-light detection and ranging for future stand-off virus surveillance," Microbial Biotechnology, 2020, doi: 10.1111/1751-7915.13698.

[24] A. K. Goyal et al., "Active infrared multispectral imaging of chemicals on surfaces," Chemical, Biological, Radiological, Nuclear, and Explosives (CBRNE) Sensing XII, vol. 8018, p. 80180N, 2011, doi: $10.1117 / 12.882698$.

[25] P. Kotidis, E. R. Deutsch, and A. Goyal, "Standoff detection of chemical and biological threats using miniature widely tunable QCLs," Micro- and Nanotechnology Sensors, Systems, and Applications VII, vol. 9467, p. 94672S, 2015, doi: 10.1117/12.2178169.

[26] E. R. Deutsch et al., "Active and passive infrared spectroscopy for the detection of environmental threats," Advanced Environmental, Chemical, and Biological Sensing Technologies XI, vol. 9106, p. 91060A, 2014, doi: 10.1117/12.2058544.

[27] C. Magdy, F. Issam, P. Amir, S. Adel, and S. Elgohary, "COVID-19 detection using SERS technique," MOJ App Bio Biomech, vol. 4, no. Figure 1, pp. 86-91, 2020, doi: 10.15406/mojabb.2020.04.00141.

[28] R. Pilot, "SERS detection of food contaminants by means of portable Raman instruments," Journal of Raman Spectroscopy, vol. 49, no. 6, pp. 954-981, 2018, doi: 10.1002/jrs.5400.

[29] E. P. Fischer, M. C. Fischer, D. Grass, I. Henrion, W. S. Warren, and E. Westman, "Low-cost measurement of facemask efficacy for filtering expelled droplets during speech," medRxiv, no. September, pp. 2-7, 2020, doi: 10.1101/2020.06.19.20132969. 